\title{
FFT based target detection using FMCW Radar
}

\author{
Rajesha K V ${ }^{1}$, Dr. U B Mahadevaswamy ${ }^{2}$ \\ ${ }^{I}$ Mtech in Industrial Electronics, SJCE, Mysuru, INDIA \\ ${ }^{2}$ Associate Professor, Department of electronics, SJCE, Mysuru, INDIA
}

\begin{abstract}
RAdio Detection And Ranging (RADAR) has been used since 1930's for the detection and estimation of target related information. Earlier Pulse radars were used to determine the target, in which there was time interval between the transmissions of pulses, which in turn induced error in determination of target. To prevent this ambiguity Continuous Wave $(C W)$ radars were used, non modulated $C W$ radar was unable to find velocity of target. So Frequency Modulated Continuous Wave (FMCW) radar is used when we have to find velocity of moving target. The purpose of this work is to develop a 1024 point FFT code for FMCW radar, through which we can convert any physical signal to frequency domain, and we are extending the same to find velocity of the target with higher precision and faster rate and also to simulate the same using FPGA.
\end{abstract}

Keywords: RADAR, FMCW, FFT, FPGA, target, Range, Velocity

\section{Introduction}

Radar is a target detection system that uses radio waves to detect target and its related information like range, angle, velocity, direction. It can be used to detect targets like flights, spacecrafts, submarines, missiles, vehicles. It is also used to find weather related information [1]. Radar transmits electromagnetic waves which are bounced back from the target in the path. The reflected waves are called echoes, which contains target related information. Electromagnetic waves travels through air at a constant speed, at approximately the speed of light, i.e. 300,000 kilometers per second. In time domain we can only know the value of the signal at a particular time, it will not provide information about the rate at which the signal is varying, and hence we have to transmit the signal in frequency domain. Fourier Transforms helps us to change a time domain signal to frequency domain [3]. In this work we are using Fast Fourier Transform for signal processing which will increase the speed of the system [4]. In Pulse radars, a series of short duration pulses are sent. A time gap is elapsed between transmissions of each pulse. Time gap is randomly calculated based on the maximum expected range of the target [6]. Pulse duration will be in micro second, whereas the time gap is in millisecond. The increased time gap leads to miss some target detection and also to increase False Alarm Ratio (FAR), which is a serious drawback of pulse radars. Continuous wave $(\mathrm{CW})$ radars were used to overcome the drawback of pulse radars. Now the challenge is to locate and find the velocity of moving targets like aircraft, missiles, etc. FMCW radar plays an important role in finding and estimating data related to moving targets [1]. FMCW radar transmits a frequency modulated continuous wave, the echo signal is time delayed and Doppler shifted version of the transmitted wave. The time delay is used to find the range of the target and Doppler shift is used to find the velocity of the target. The signal to noise ratio is highly improved in FMCW radar compared to pulse radars, and false alarm rate is reduced in FMCW radar.

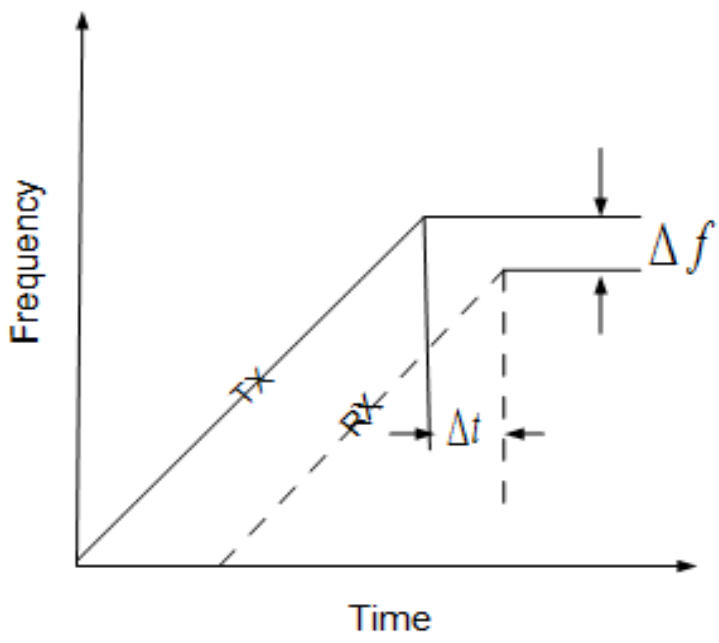

Figure 1: Time vs. Frequency plot of transmitted and received signal in FMCW radar. 
The target related information like Range can be estimated using Equation 1 and velocity can be found by using Equation 2 by obtaining the required parameters from the received radar signal.

$$
\text { Range }=\frac{C|\Delta t|}{2}=\frac{C|\Delta f|}{2\left(\frac{d f}{d t}\right)}
$$

Where:

$\mathrm{C}=$ speed of light $=3 * 10^{8} \mathrm{~m} / \mathrm{s}$

$\Delta \mathrm{t}=$ propagation delay $[\mathrm{s}]$

$\Delta \mathrm{f}=$ frequency difference $[\mathrm{Hz}]$

$\mathrm{df} / \mathrm{dt}=$ frequency shift per unit of time

In the proposed work as we are not working on real time radar, the time taken by the transmitted signal to return to the radar is unknown; hence we are not finding the range of the target.

Where:

$$
\text { velocity }=\frac{C|\Delta f|}{2 f_{t}}
$$

$\mathrm{f}_{\mathrm{t}}=$ transmitted signal frequency

To find velocity of the target it is not possible without converting signal in frequency domain, in this work we develop a VHDL code for 1024 point FFT using IP cores of Quartus II, so that we can readily use it in DSP chips. And we assume that the received signal is 10 percent frequency shifted transmitted signal and the velocity of the target was found.

\section{Implementation}

Here we are going to discuss about the design flow of software implementation and block diagram of hardware implementation

\section{Software implementation}

We have used both MATLAB and Quartus II altera in our work, MATLAB is used for simulation purpose, but Quartus II software easily adapts to the specific design needs. It includes solutions for all phases of FPGA and CPLD design. We use mega functions and IP functions of Quartus II to instantiate our design [13]. Figure 2 shows design flow of developing 1024 point FFT code using MATLAB and Quartus II, the design flow is done in a preset hierarchy and is clearly explained in the flowchart. The 1024 point FFT code was first realized in MATLAB without using the inbuilt FFT function, but MATLAB is not a hardware descriptive language, hence further the same code was realized in hardware description language in Quartus II altera, so that it can be used on FPGA.

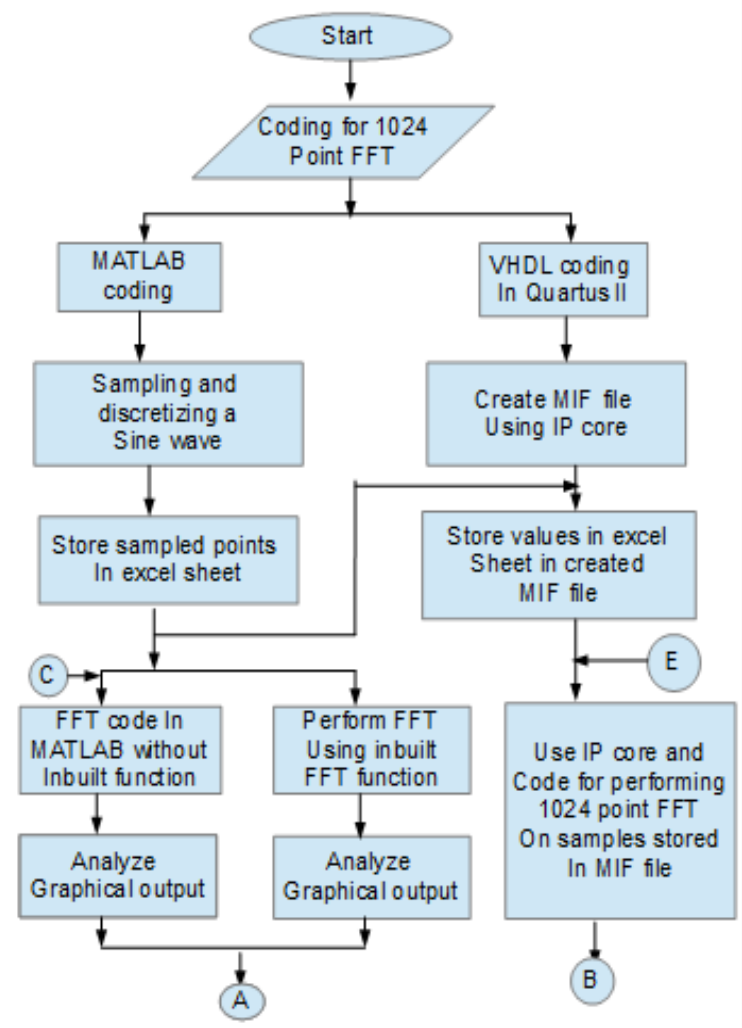



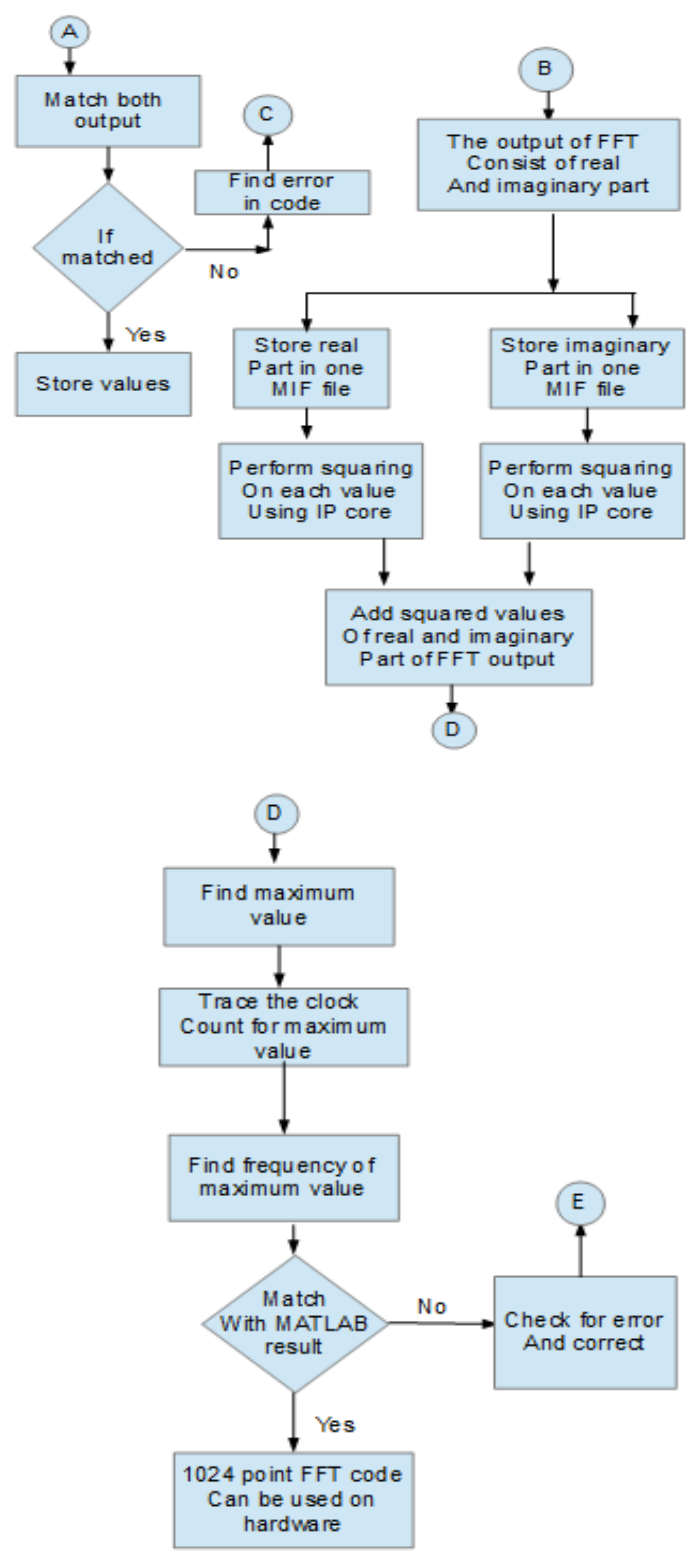

Figure 2: Design flow of developing 1024 point FFT code using MATLAB and Quartus II.

\section{Hardware implementation}

The block diagram of proposed methodology is shown in the Figure 3; it consists of two major blocks

A. Host machine

B. FPGA

Host machine is the one through which we can build our design with the help of software, it may be PC, laptop, tablet, which is compatible for the usage of software. The output is examined with the help of host machine.

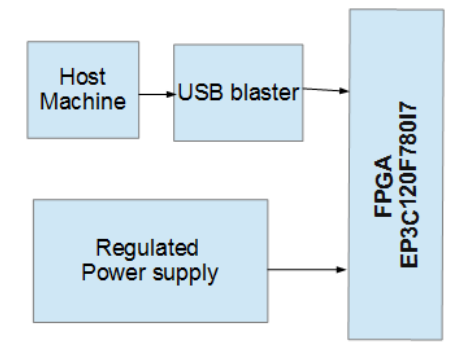

Figure 3: Block diagram of implementation 
The FPGA used in this proposed work is EP3C120F780I7 (Cyclone III device family by altera), it's a package of 780 pins and having a speed grade 7 [12]. Its operating temperature range varies from -40 to 100 degree Celsius. It is operated with 5 Volts, 2 Ampere regulated power supply. USB blaster is used to interface host machine with FPGA, the program is loaded to FPGA with the help of USB blaster. And regulated power supply provides the required voltage and current for FPGA

\section{Results}

We have tapped all the required input and output signals provided to FPGA in Signal Tap II Logic Analyzer of Quartus II altera. The main output signals like Doppler shift, received frequency, velocity can be observed in figure 4 and figure 5.The result shown is for received signal which is 10 percent frequency shifted transmitted signal, processed with 1024 point FFT. If the received signal frequency is more than transmitted signal frequency, then the target is travelling towards the radar. If the received signal frequency is less than the transmitted signal frequency, then the target is moving away from the radar. In Figure 4, transmitted signal frequency is $100 \mathrm{~Hz}$, but in Figure 5, transmitted signal frequency is $1000 \mathrm{~Hz}$.

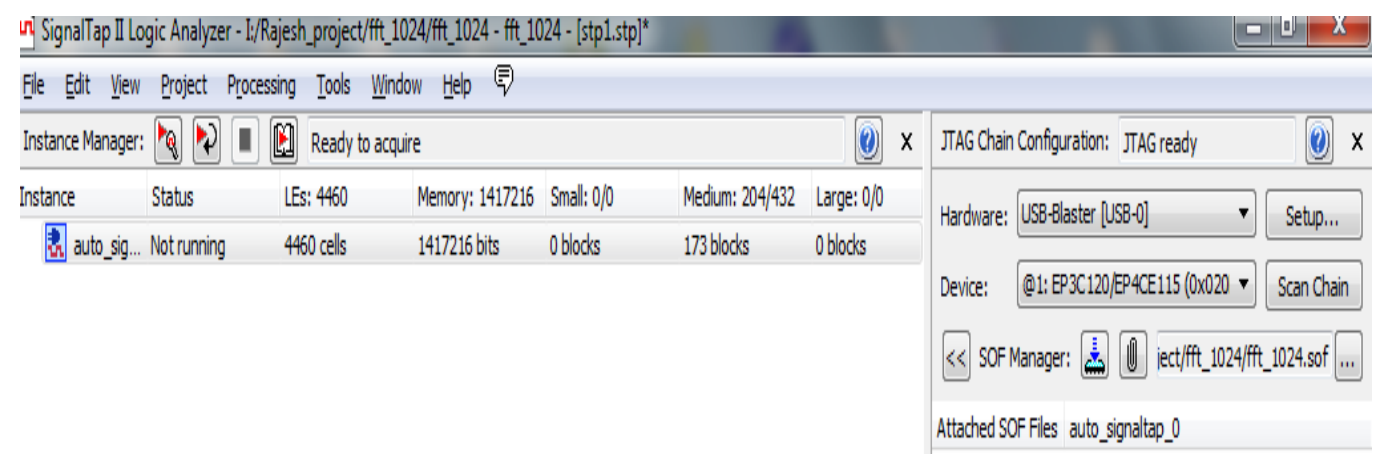

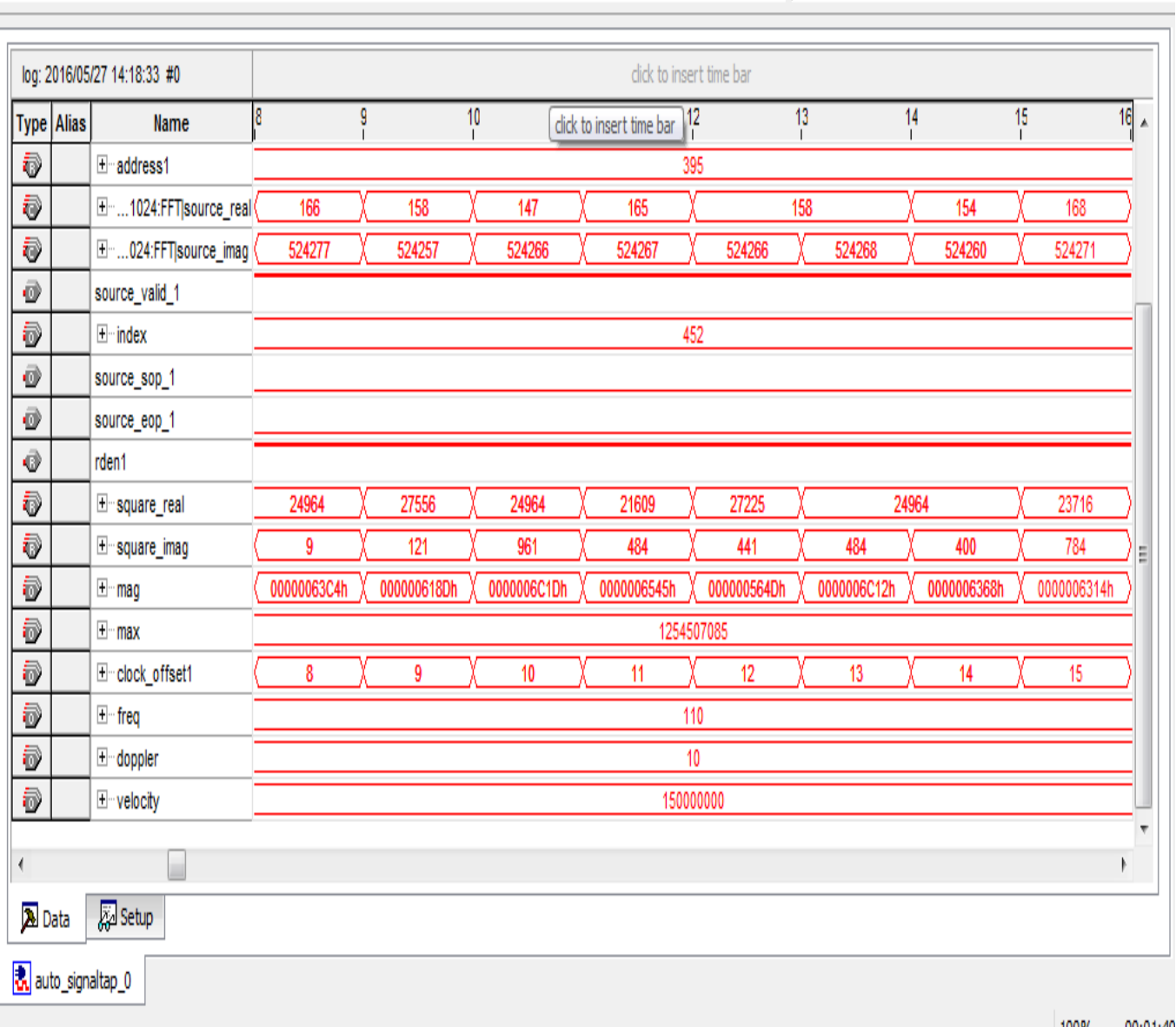

Figure 4. Signal Tap II Logic Analyzer output for $100 \mathrm{~Hz}$ transmitted freq and $110 \mathrm{~Hz}$ received frequency 


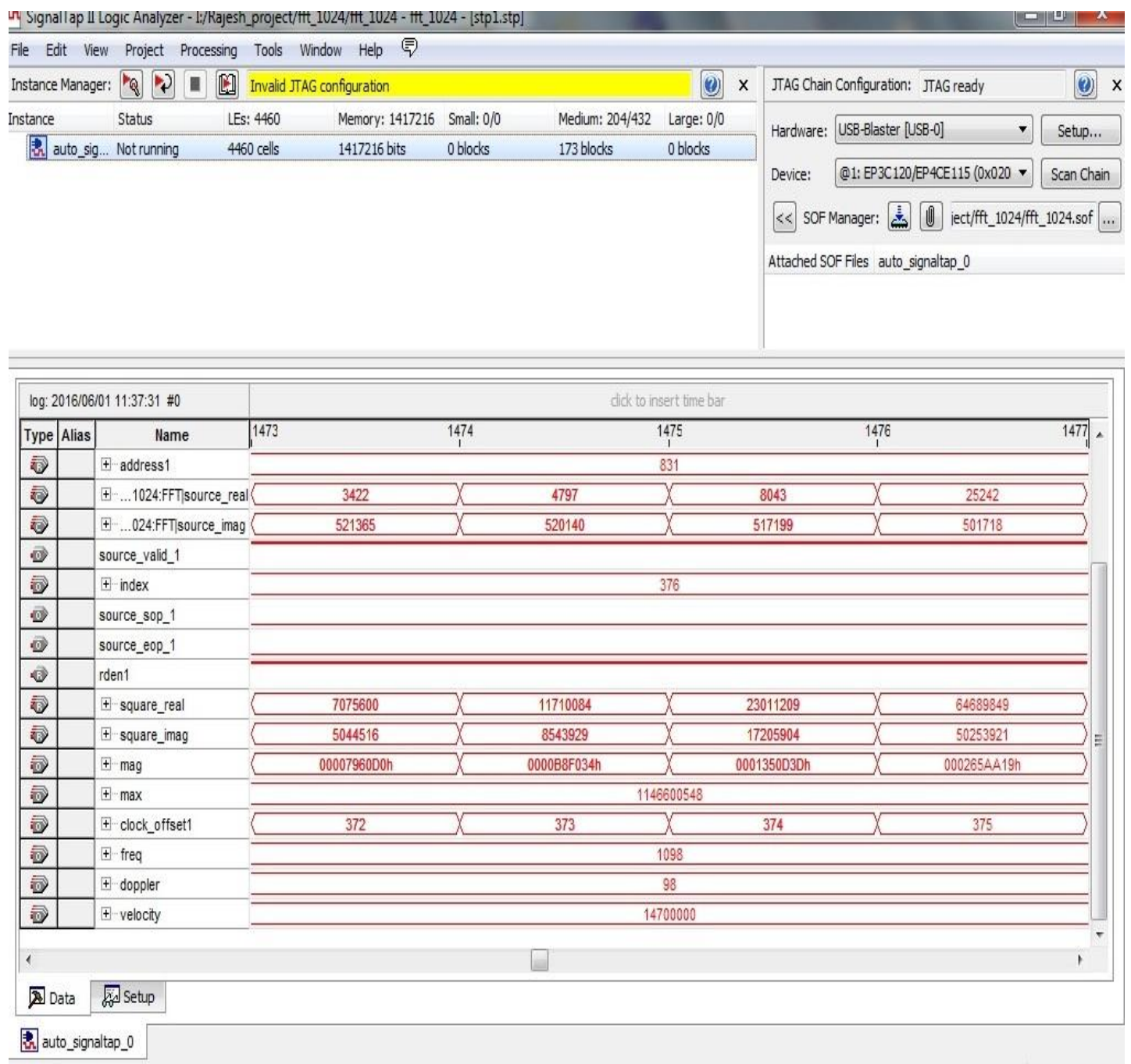

Figure 5. Signal Tap II Logic Analyzer output for $1000 \mathrm{~Hz}$ transmitted and $1100 \mathrm{~Hz}$ received frequency

The velocity in both figure 4 and figure 5 should be same, as Doppler shift is 10 percent. The calculated velocity is 15000000 , but we can observe that there is error in detection of velocity in Figure 5, that is because variation in delta.

$$
\text { delta }=\frac{f_{t}}{N}
$$

Where:

$\mathrm{f}_{\mathrm{t}}=$ transmitted signal frequency

$\mathrm{N}=$ number of FFT points.

\section{Conclusion and future enhancement}

The solution was successfully implemented using fixed point processing. Here we have to compromise between transmitted signal frequency and number of FFT points to have better results. The solution can also be implemented using single or double precision Floating point which will lead us towards more accurate results.

The work is carried out for single dimension (1D), we can also implement this work for three dimensional radar (3D). And new method other than FFT should be proposed to enhance the speed of the computation. The work was carried out for ideal condition in which external factors like noise was not considered, proper filters should be proposed if the work is carried in non ideal condition.

\section{Reference}

[1]. M. I. Skolnik, Introduction to radar systems, 3rd ed. Mc- Graw-Hill Ltd., 2001.

[2]. Automotive Radar by Silicon-Germanium Technology by D.Kissinger, DOI 10.1007/978-1-4614-2290-7 2, (C) Springer Science and Business Media, LLC 2012.

[3]. Signal and systems by Simon haykin, copyright 1999, john Wiley and sons.

[4]. The Fast Fourier Transform and Its Applications by James W. Cooley, Peter A. W. Lewis, and Peter D. Welch, IEEE transactions on education, vol. 12, no. 1, march 1969. 
[5]. Implementation of a 128-Point FFT on the MRC6011 Device by Zhao Li, Hirokazu Higa, and Ed Martinez, (C) Freescale Semiconductor, Inc., 2004.

[6]. Radar System Technologies by S.Varadarajan, Defense Research and Development Organization, Vol. 21 No. 2 April 2013, ISSN: 0971-4413.

[7]. Detection Performance Improvement of FMCW Radar Using Frequency Shift, by Yan Wu and J P Linnartz, WIC/IEEE SP Symposium on Information Theory and Signal Processing in the Benelux, Brussels, Belgium, May 10-11, 2011.

[8]. Two-dimensional signal processing in FMCW radars by Andrzej Wojtkiewicz, Jacek Misiurewicz, Marek Nałe, cz Konrad Je, drzejewski and Krzysztof Kulpa, Instytut Podstaw Elektroniki,2011.

[9]. Waveform Design Principles for Automotive Radar Systems by Hermann Rohling, Marc-Michael Meinecke, Technical University of Hamburg-Harburg/ Germany, 2001.

[10]. Understanding Millimeter Wave FMCW Radars by Graham M Brooker, 1st International Conference on Sensing Technology November 21-23, 2005 Palmerstone North, New Zealand.

[11]. Sampling of continuous-time signals by J. Carwardine, USPAS 2003.

[12]. Cyclone 3 device handbook, Copyright @ 2010 Altera Corporation

[13]. Introduction to the Quartus ${ }^{\circledR}$ II Software, version 10.0, altera.com

[14]. L. Ding, M. Ali, S. Patole and A. Dabak, "Vibration parameter estimation using FMCW radar," 2016 IEEE International Conference on Acoustics, Speech and Signal Processing (ICASSP), Shanghai, 2016, pp. 2224-2228.

[15]. Y. Kim, "Identification of FMCW radar in mutual interference environments using frequency ramp modulation," 2016 10th European Conference on Antennas and Propagation (EuCAP), Davos, 2016 